\title{
Random Early Blocking with Engineered Threshold in Wireless Multimedia Networks
}

\author{
Shun-Ping Chung and Jin-Chang Lee \\ Department of Electrical Engineering \\ National Taiwan University of Science and Technology \\ Taipei, Taiwan, R.O.C.
}

\begin{abstract}
These Driven by the idea of integrating all services, such as voice, data, video, and multimedia into the wireless domain, related topics have been on the horizon. A novel Random Early Blocking with Engineered Threshold (REB-ET) CAC scheme, taking account of the concept of virtual partitioning as well as acceptance probability, is proposed to achieve high resource utilization as well as maintain the blocking probability at an acceptable level in wireless multimedia network with conversational, streaming and background services. An analytical method is developed to calculate performance measures of interest, i.e., new call blocking probability and forced termination probability for conversational and streaming services, average available bandwidth for streaming services, and average packet delay for background services. It is shown that analytical results are close to the simulation results. Also, numerical results under not only stationary but also nonstationary scenario are shown to illustrate the robustness of the proposed scheme.
\end{abstract}

\section{INTRODUCTION}

It is well known that the UMTS/IMT2000 is supposed to be a multiservice network providing QoS guarantee to four classes of services: conversational, streaming, interactive, and background. Thus, for such a multiservice network, it is imperative for CAC to make efficient use of scarce wireless resource while keeping the quality of service $(\mathrm{QoS})$ at an acceptable level. Furthermore, forced termination of calls are more annoying than blocking new calls in progress. Handoff calls are usually given priority over new calls, e.g., with guard channels or trunk reservation (TR), but at the expense of lower channel utilization. It is worth noting that handoff call arrival rate depends strongly on call holding time and call holding time difference among different services may be significant. It is pointed out that the average call holding time of voice calls is $3 \mathrm{~min}$, while that of data calls, e.g., Internet browsing, is in the range of $20 \mathrm{~min}$ [1]. This call holding time difference may result in serious impact on the performance measures of interest in wireless multimedia network with different service classes.

A novel CAC, random early blocking with engineered threshold (REB-ET), taking account of the concept of virtual partitioning [2] as well as acceptance probability [3], is proposed for multiservice wireless networks with conversational, streaming and background services. An analytical method is developed to calculate performance measures of interest, i.e., new call blocking probability and forced termination probability for conversational and streaming services, average available bandwidth for streaming services, and average packet delay for background services. Last but not least, the performance measures of interest under not only stationary but also nonstationary traffic load are studied.

The remainder of this paper is organized as follows. In Section 2, the associated system model and the proposed REB-ET are described. The analytical method is described in detail in Section 3, and numerical results are shown in Section 4. Some concluding remarks are given in the last section.

\section{SYSTEM MODEL}

A TDMA/TDD based protocol is adopted to support streaming, streaming, and background services. Assume that each fixed length frame is divided into $C=C_{1}+C_{2}$ time slots, where $C_{1}$ time slots are shared among all services, and $C_{2}$ time slots are available to background service exclusively. The conversational services have constant bandwidth requirement. The streaming services have minimum and maximum bandwidth requirements. More specifically, conversational services with higher priorities are assigned time slots periodically based on their bandwidth requirements, streaming services are assigned slots dynamically based on the usage of bandwidth resource, and the background services with lowest priority use the remaining time slots. It is assumed that background service is provided with an infinite queue so that it is always accepted regardless of the number of time slots used by conversational and streaming services. It is noted that in this paper conversational services are associated with short call holding time, such as telephone and videophone, streaming services with longer call holding time, such as adaptive multimedia, and background services with shorter call holding time, such as e-mail.

Assume that there are $K$ classes of conversational services, streaming services and background services. The new and handoff arrival processes of conversational services are assumed to be Poisson. The call holding time and the dwell time of conversational services are assumed to follow an exponential distribution. The new call and the handoff arrival process of streaming service are assumed to be Poisson. Furthermore, the call holding time and the dwell time of streaming services are exponentially distributed. The streaming service is taken as the class- $(K+1)$ service for easy explanation. For background services, the packet arrival process is assumed to be Poisson, and the packet service time is exponentially distributed with mean service rate depending on the number of time slots available. Some parameters used in the following are defined in Table 1. 
Table 1. Some parameters for the focused system

\begin{tabular}{|c|c|}
\hline$C$ & $\begin{array}{l}\text { the number of time slots per frame allocated to the focused } \\
\text { cell }\end{array}$ \\
\hline$C_{1}\left(C_{2}\right)$ & the number of time slots for all (background) services \\
\hline$T_{k}\left(T_{K+1}\right)$ & threshold of class- $k$ conversational (streaming) services \\
\hline$\lambda_{n k}\left(\lambda_{n K+1}\right)$ & $\begin{array}{l}\text { new call arrival rate of class- } k \text { conversational (streaming) } \\
\text { services }\end{array}$ \\
\hline$\lambda_{h k}\left(\lambda_{h K+1}\right)$ & $\begin{array}{l}\text { handoff call arrival rate of class- } k \text { conversational services } \\
\text { (streaming) services }\end{array}$ \\
\hline$b_{k}$ & time slot requirement of class- $k$ conversational services \\
\hline$b_{P}\left(b_{K+1}\right)$ & $\begin{array}{l}\text { maximum and minimum time slot requirements of } \\
\text { streaming services }\end{array}$ \\
\hline$b_{A}$ & average number of assigned time slots of streaming services \\
\hline$\mu_{k}\left(\mu_{K+1}\right)$ & $\begin{array}{l}\text { call service rate of class- } k \text { conversational (streaming) } \\
\text { services }\end{array}$ \\
\hline$\eta_{k}\left(\eta_{K+1}\right)$ & dwell rate of class- $k$ conversational (streaming) services \\
\hline$n_{k}\left(n_{K+1}\right)$ & $\begin{array}{l}\text { the number of ongoing class- } k \text { conversational (streaming) } \\
\text { services }\end{array}$ \\
\hline$n_{\min }$ & $\begin{array}{l}\text { the total number of minimum channels occupied by } \\
\text { conversational and streaming services }\end{array}$ \\
\hline$\lambda_{U}$ & new call arrival rate of background services \\
\hline
\end{tabular}

\section{A. Random Early Blocking with Engineered Threshold}

One key idea of REB-ET is that a service-dependent engineered threshold should be determined based on bandwidth and QoS requirements, such that new call blocking probability is below some acceptable level for the engineered (expected) load, and thus resource utilization is above some acceptable level. There are several methods to find optimal value of the number of guard channel for handoff calls [4]. We try to find an efficient way to determine a suboptimal threshold via an empirical formulation. With virtual partitioning (VP) in [2], if the bandwidth occupied by class- $k$ services is below its nominal capacity $N_{k}$, a newly arriving class- $k$ service will be admitted as long as there is enough bandwidth available. On the other hand, if the bandwidth occupied by class- $k$ services exceeds its nominal capacity $N_{k}$, a newly arriving class- $k$ service will be admitted only if the free bandwidth is larger than a class-specific trunk reservation. Furthermore, the relationship of the nominal capacity $N_{k}$, the bandwidth requirement $b_{k}$, and the engineered load $\rho_{E N G, k}$ is as follows.

$$
\rho_{E N G, k}=\frac{N_{k}}{b_{k}}-\sqrt{\frac{N_{k}}{b_{k}}}
$$

Specifically, if the offered load $\rho_{k}=\lambda_{k} / \mu_{k} \leq \rho_{E N G, k}$, the blocking probability of class- $k$ services is below some acceptable level.

We generalize the idea of VP to compute the suboptimal engineered threshold for each class of conversational and streaming services. Since the average number of assigned time slots to streaming services, $b_{A}$, depends on the system state, for easy calculation of the nominal capacity of streaming services, we will approximate $b_{A}$ with the average of $b_{P}$ and $b_{K+1}$ in the derivation of engineered threshold. Let the total engineered load to each cell due to new calls be denoted as $\gamma_{T}$, including conversational and streaming services. There are two differences between VP and the proposed ET. First, to take account of handoff calls due to mobility, we set $\gamma_{T}=\phi C_{1}$. Second, unlike virtual partitioning, if $n_{\min }$ is less than $T_{k}$, the newly arriving class- $k$ call is admitted as long as there are enough bandwidth available. Therefore, we should use $\gamma_{T} / b_{k}$ in place of $\rho_{E N G, k}$ in calculating the threshold $T_{k}$. Furthermore, according to our extensive study, it is found that $\phi=0.65$ can achieve overall better results. To summarize, the threshold $T_{k}$ of class- $k$ conversational service is computed as follows.

$$
T_{k}=\min \left\{C_{1},\left\lfloor b_{k}\left(1+\frac{\sqrt{1+\left(2.6 \mathrm{C}_{1} / b_{k}\right)}}{2}\right)^{2}\right\}\right\}
$$

It is noted that the threshold $T_{K+1}$ of streaming service is derived with $b_{k}$ being replaced by the average of $b_{P}$ and $b_{K+1}$ in the above equation.

Furthermore, instead of blocking any new call attempting to utilize guard channels as TR, REB-ET takes account of the idea of acceptance probability for new calls. That is, the guard channels, reserved preferentially for handoff calls, can be allocated to new calls based on some acceptance probabilities. Let $A_{k}\left(n_{\min }\right)$ be the acceptance probability of class- $k$ services. After extensive numerical study and with reference to [3], $A_{k}\left(n_{\min }\right)$ is defined as follows:

$$
A_{k}\left(n_{\text {min }}\right)=\operatorname{Max}\left\{0, f_{k}\left(n_{\text {min }}\right)\right\} \text {, }
$$

where $f_{k}\left(n_{\min }\right)$ is calculated as follows:

$$
f_{k}\left(n_{\min }\right)=\left\{\begin{array}{l}
\frac{\eta_{k}}{\mu_{k}}\left[\frac{\left(C_{1}-b_{k}+1\right)-n_{\min }}{\left(C_{1}-b_{k}+1\right)-T_{k}}\right] \\
+\left(1-\frac{\eta_{k}}{\mu_{k}}\right) \times\left[\cos \frac{2 \pi\left(n_{\min }-T_{k}\right)}{4\left(\left(C_{1}-b_{k}+1\right)-T_{k}\right)}\right]^{1 / 2}, n_{\min }>T_{k}
\end{array}\right.
$$

When $n_{\text {min }}$ is greater than $T_{k}$, the remaining channels can also be allocated to new calls according to $A_{k}\left(n_{\text {min }}\right)$ based on $\eta_{k} / \mu_{k}$. More specifically, if $\eta_{k}>\mu_{k}$, it implies that on average more than one handoff event would occur during one average call holding time, CAC should make more room for handoff calls, such that lower forced termination probability can be achieved. If $\eta_{k}=\mu_{k}, A_{k}\left(n_{\text {min }}\right)$ can be chosen to decrease linearly with the number of available channels. On the other hand, if $\eta_{k}<\mu_{k}$, CAC should allow a higher probability in accepting new calls for achieving lower new call blocking probability, while maintaining an acceptable forced termination probability for handoff calls. 


\section{B. Bandwidth Allocation}

If the total number of minimum channels occupied by existing conversational and streaming services is less than $C_{1}$, the newly arriving class- $k$ conversational call could be admitted based on $A_{k}\left(n_{\text {min }}\right)$. If the newly arriving conversational call is accepted, the time slots assigned to existing streaming services will be reallocated to accommodate the incoming conversational call if it is necessary. For fairness purposes, besides the time slots occupied by conversational services, the remaining time slots of $C_{1}$ are equally shared among the streaming services in progress in a memoryless fashion. Similarly, a newly arriving streaming call will also be admitted based on $n_{\min }$ and $A_{K+1}\left(n_{\text {min }}\right)$. It is also noted that bandwidth reallocation of streaming services are enforced if needed whenever one conversational or streaming call is complete. Additionally, for reducing forced termination probability, a handoff class- $k$ conversational (streaming) call will be accepted as long as there are enough time slots, i.e., $n_{\min }$ plus $b_{k}\left(b_{K+1}\right)$ is less than or equal to $C_{1}$, in the target cell at the arrival time of handoff conversational (streaming) services.

The background service is always admitted, and waits in queue if no time slot is available at the time of request. In addition to $C_{2}$ time slots, the background service can use any unused time slots in $C_{1}$.

\section{ANALYTICAL METHOD}

For simplicity, we consider a homogeneous cellular network with FCA, and thus can focus on one particular cell. Because of the lower priority of background services, the analysis of background service can be dealt with separately.

The associated $(K+1)$-dimensional Markov chain of the model under study is now described. The state vector is $\vec{n}=\left(n_{1}, n_{2}, \ldots n_{K+1}\right)$. Let $\vec{n}_{k}^{-}=\left(n_{1}, n_{2}, \ldots, n_{k}-1, \ldots, n_{K+1}\right)$ and $\vec{n}_{k}^{+}=\left(n_{1}, n_{2}, \ldots, n_{k}+1, \ldots, n_{K+1}\right)$. The feasible state space $S:=\left\{\vec{n} \in \mathrm{IN}^{K+1}: \sum_{l=1}^{K+1} b_{l} n_{l} \leq C_{1}\right\}$. The state transition diagram for an example cell with two classes of conversational services and one class of streaming services is shown in Fig. 1.

The steady state probability $\pi(\vec{n})$ can be obtained via a Gauss-Seidel algorithm by solving the state equations of the considered system. It is noted that the handoff arrival rate for each service is computed as follows:

$$
\lambda_{h k}=\left(1-P_{h b k}\right) P_{h k} \lambda_{h k}+\left(\sum_{n_{\min }=0}^{C_{1}} A_{k}\left(n_{\min }\right) \pi\left(n_{\min }\right)\right) \lambda_{n k} P_{h k}
$$

where $P_{h b k}=\sum_{n_{\min }=C-b_{k}+1}^{C_{1}} \pi\left(n_{\min }\right)$ and $P_{h k}=\frac{\eta_{k}}{\mu_{k}+\eta_{k}}$. where $\pi\left(n_{\text {min }}\right)$ is the steady state probability that the total number of minimum channels occupied by conversational and streaming services is $n_{\min }$.

The blocking probability of new class-k conversational services can be determined as follows:

$$
P_{b k}=\sum_{n_{\min }=T_{k}}^{C_{1}}\left[1-A_{k}\left(n_{\min }\right)\right] \pi\left(n_{\min }\right)
$$

Furthermore, the forced termination probability of handoff class-k conversational services can be computed as follows:

$$
P_{f k}=\frac{P_{h k} P_{h b k}}{\left[1-P_{h k}\left(1-P_{h b k}\right)\right]}
$$

Second, the analysis of streaming services is described. As to the number of time slots assigned to one streaming call, there are two cases. One case is that $b_{p}$ time slots are assigned to all streaming services, when the number of ongoing streaming services is no greater than $\beta=\left\lfloor C_{1}-\sum_{l=1}^{K} b_{l} n_{l} / b_{P}\right\rfloor$. The other case is that the number of ongoing streaming services is greater than $\beta$, and thus the number of time slots assigned to each streaming call may not be equal to $b_{p}$ and may differ by one. The average number of assigned time slots of streaming services can be compute as follows:

$$
\begin{aligned}
& b_{A}=\sum_{\vec{n} \in S, n_{K+1}=0}^{\beta} b_{P} \pi(\vec{n})+\sum_{\vec{n} \in S, n_{K+1}=\beta+1}^{\left\lfloor b_{b+1}\right\rfloor} \alpha \pi(\vec{n}) \\
& \text { where } \alpha=\left(C_{1}-\sum_{l=1}^{K} b_{l} n_{l}\right) / n_{K+1} .
\end{aligned}
$$

The blocking probability of new streaming services can be determined as follows.

$$
P_{b K+1}=\sum_{n_{\min }=T_{K+1}}^{C}\left[1-A_{K+1}\left(n_{\min }\right)\right] \pi\left(n_{\min }\right)
$$

Furthermore, the forced termination probability of handoff streaming services can be computed as follows:

$$
P_{f K+1}=\frac{P_{h K+1} P_{h b K+1}}{\left[1-P_{h K+1}\left(1-P_{h b K+1}\right)\right]}
$$

Lastly, the analysis of background services is described. Suppose that the average durations of conversational and streaming services are much longer than the packet transmission time of background service. Thus, the bandwidth used by conversational and streaming services can be viewed as constant by background service. An $\mathrm{M} / \mathrm{M} / 1$ queue is used to determine the average packet delay of background services in the following.

$$
E\left[T_{d}\right]=\sum_{\vec{n} \in S} E\left[T_{d} \mid \vec{n}\right] \pi^{\text {new }}(\vec{n})
$$

where $E\left[T_{d} \mid \vec{n}\right]$ is computed via the following identity:

$$
E\left[T_{d} \mid \vec{n}\right]= \begin{cases}\frac{\lambda_{u}}{\left(C-\sum_{l=1}^{K} b_{l} n_{l}-b_{P} n_{K+1}\right)\left[\left(C-\sum_{l=1}^{K} b_{l} n_{l}-b_{P} n_{K+1}\right)-\lambda_{u}\right]} & n_{K+1} \leq \beta \\ \frac{\lambda_{u}}{C_{2}\left(C_{2}-\lambda_{u}\right)}, & n_{K+1}>\beta\end{cases}
$$




\section{NUMERICAL RESULTS}

A cellular wireless multiservice network with two kinds of conversational, streaming and background services is studied. Some of the parameters used are $C_{1}=60, C_{2}=5$, $b_{1}=1,1 / \mu_{1}=180 \mathrm{sec}, b_{2}=4,1 / \mu_{2}=300 \mathrm{sec}, b_{P}=5, b_{3}=1$ and $1 / \mu_{3}=1200 \mathrm{sec}$. Fixed trunk reservation (TR) is also considered for comparison. In TR, the number of reserved channels for handoff calls exclusively is denoted as $\mathrm{g}$, and three cases with $\mathrm{g}=0,9$, and 12 are studied. With REB-ET, the thresholds for class-1 conversational new calls, class-2 conversational new calls, streaming new calls, which are determined based on ET, are 45, 53 and 51, respectively. The QoS requirements of conversational services are (1) the new call blocking probability is smaller than 0.02 , and (2) the forced termination probability is smaller than 0.002 . Moreover, the QoS requirement of background service is that average packet delay is smaller than $0.06 \mathrm{sec}$. The QoS requirements of streaming service are the same as those of conversational service. Due to lack of space, some results are not shown.

First of all, simulation results are shown to verify the accuracy of analytical results. The parameters used are $\lambda_{n 1}=0.13333, \lambda_{n 3}=0.00167$, and $\lambda_{u}=3.6$. Further, $\lambda_{n 2}$ is varied from 0.005 to 0.010 in steps of size 0.001 . The analytical and simulation results for new call blocking probability are shown in Figs. 2. As can be seen, the analytical results are very close to the simulation results. Similar conclusions can be reached for forced termination probability and average number of assigned time slots of streaming service.

The robustness of REB-ET is demonstrated in the following. The parameters used are the same as those in Fig. 2. Some numerical results for TR and REB-ET are shown in Figs. 3 to 4 . It is found that both the new call blocking and forced termination probabilities of conversational and streaming calls increase as the new call arrival rate of class- 2 conversational calls increases. The higher offered load of class-2 conversational calls also results in a decrease of average number of assigned time slots of streaming calls as well as an increase of average packet delay of background calls. Obviously, the more time slots occupied by conversational calls with higher priority, the fewer time slots available to streaming and background calls. It is also found that $\operatorname{TR}(g=0)$ with $\lambda_{n 2}<0.07, \operatorname{TR}(g=9)$ with $\lambda_{n 2}<0.06$, $\operatorname{TR}(g=12)$ with $\lambda_{n 2}<0.05$ and REB-ET with $\lambda_{n 2}<0.09$ can satisfy aforesaid QoS goals. Moreover, in terms of the average number of assigned time slots of streaming calls, REB-ET outperforms $\operatorname{TR}(g=6), \operatorname{TR}(g=9)$, and $\operatorname{TR}(g=12)$. On the other hand, it can be found that the new call blocking probabilities with $\operatorname{TR}(g=0)$ are smaller, but $\operatorname{TR}(g=0)$ also results in higher forced termination probabilities.

Last but not least, the nonstationary scenarios are studied via computer simulation. Under nonstationary scenarios, each cell alternates in two states: busy and normal, independently of one another. A cell in the busy state is assumed to have higher new call arrival rate than that in the normal state, and thus slower velocity and longer dwell time due to increasing congestion. The average speed of the mobile stations in the normal (busy) state is taken to be $V=3.6(2.8) \mathrm{km} / \mathrm{hr}$. The duration of each state is exponentially distributed. The transition rate from normal to busy (from busy to normal) is $1 / 1800 \mathrm{sec}(1 / 300 \mathrm{sec})$. In the normal state, the parameters used are $\lambda_{n 1}=0.1, \lambda_{n 2}=0.006, \lambda_{n 3}=0.002$, and $\lambda_{u}=3.6$. To study the effect of new call arrival rates, the new call arrival rates of conversational and streaming calls in the busy state are varied from 1.5 times to 2.0 times that in normal state with steps of size 0.1. The results are shown in Figs. 5 to 6 . For easy explanation, the ratio of new call arrival rate in the busy state to that in the normal state is defined as $\varphi$. From the results, it is found that $\mathrm{TR}(\mathrm{g}=0)$ with $\varphi<1.7, \mathrm{TR}(\mathrm{g}=9)$ with $\varphi<1.7, \operatorname{TR}(\mathrm{g}=12)$ with $\varphi<1.5$ and REB-ET with $\varphi<2.0$ can satisfy aforesaid QoS requirements.

\section{CONCLUSION}

A novel Random Early Blocking with Engineered Threshold (REB-ET) CAC scheme is proposed to achieve high resource utilization as well as maintain the forced termination probability at an acceptable level in wireless multiservice network with multiple conversational services, streaming service and background service. An analytical method is derived to compute the performance measures of interest. It is shown that analytical results are very close to simulation results. It is also shown REB-ET is robust compared to fixed trunk reservation.

\section{REFERENCES}

[1] M. Cheng and L. F. Chang, "Wireless Dynamic Channel Assignment Performance under Packet Data Traffic," IEEE JSAC, vol. 17, no. 7, 1999, pp. $1257-1269$.

[2] S. C. Borst and D. Mitra, "Virtual Partitioning for Robust Resource Sharing: Computational Techniques for Heterogeneous Traffic," IEEE JSAC, vol. 16, no. 5, 1998, pp. 668-678.

[3] Y. C. Kim, D. E. Lee, B. J. Lee, Y. S. Kim, and B. Mukherjee, "Dynamic Channel Reservation Based on Mobility in Wireless ATM," IEEE Comm. Mag., vol. 37, no. 11, 1999, pp.47-51.

[4] R. Ramjee, R. Nagarajan and D. Towsley, "On Optimal Call Admission Control in Cellular Networks," Proc. IEEE INFOCOM'96, 1996, pp. 4350.

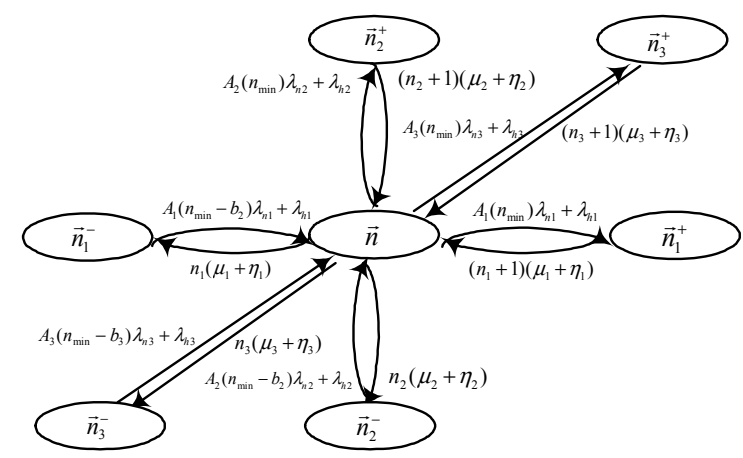

Fig. 1: State transition diagram for the example cell 


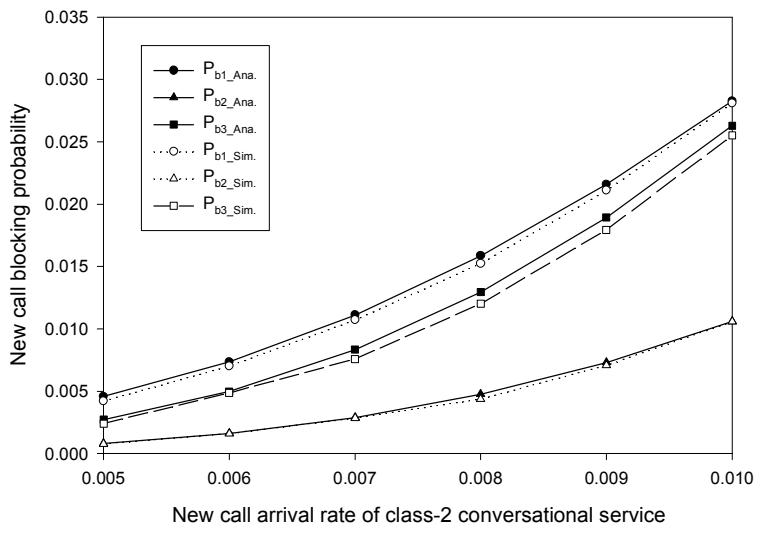

Fig. 2: New call blocking probability vs. new call arrival rate of class-2 conversational service

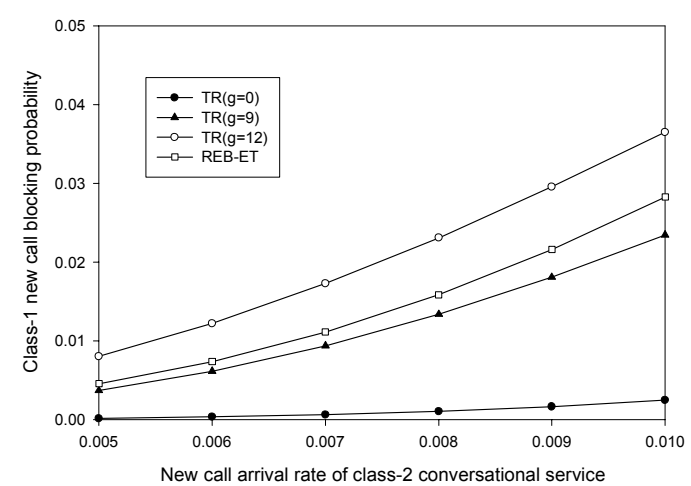

Fig. 3: Class-1 new call blocking probability vs. new call arrival rate of class2 conversational service

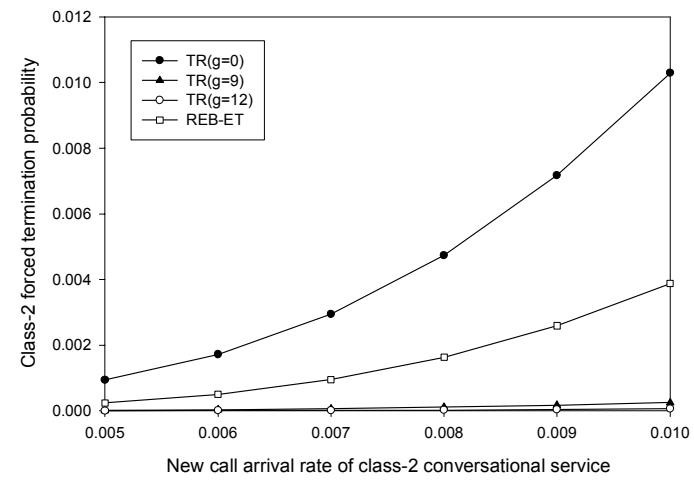

Fig. 4: Class-2 forced termination probability vs. new call arrival rate of class-2 conversational service

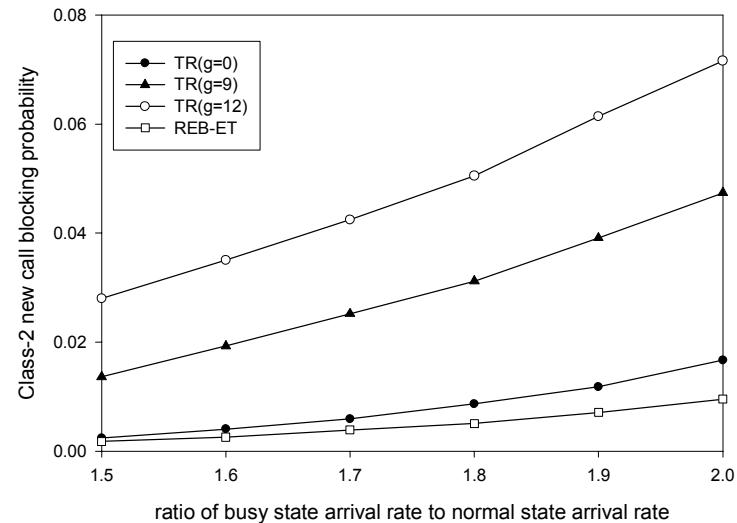

Fig. 5: Class-2 new call blocking probability vs. ratio of busy state arrival rate to normal state arrival rate

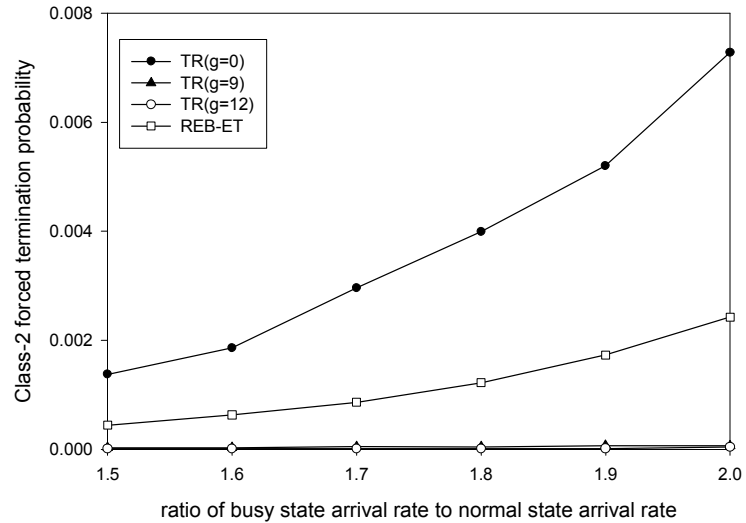

Fig. 6: Class-2 forced termination probability vs. ratio of busy state arrival rate to normal state arrival rate 